\title{
Multiple pulmonary ground glass opacities: is it time for new guidelines?
}

\author{
Alan D. L. Sihoe ${ }^{1,2}$, Rene H. Petersen ${ }^{3}$, Giuseppe Cardillo ${ }^{4}$ \\ ${ }^{1}$ Department of Surgery, The University of Hong Kong, Hong Kong, China; ${ }^{2}$ Department of Surgery, The University of Hong Kong Shenzhen \\ Hospital, Shenzhen 518053, China; ${ }^{3}$ Department of Cardiothoracic Surgery, Copenhagen University Hospital, Rigshospitalet, Copenhagen, \\ Denmark; ${ }^{4}$ Unit of Thoracic Surgery, Azienda Ospedaliera San Camillo Forlanini, Rome, Italy \\ Correspondence to: Alan D. L. Sihoe. Department of Surgery, The University of Hong Kong, Hong Kong, China. Email: adls1@lycos.com.
}

Submitted Oct 11, 2018. Accepted for publication Oct 17, 2018.

doi: $10.21037 /$ jtd.2018.10.67

View this article at: http://dx.doi.org/10.21037/jtd.2018.10.67

The National Lung Screening Trial (NLST) enrolled 53,454 current or former heavy smokers aged 55 to 74 years, and showed a relative reduction in mortality from lung cancer with low-dose computed tomography (CT) screening of $20.0 \%$ [ $95 \%$ confidence interval (CI), 6.8 to 26.7; $\mathrm{P}=0.004]$ (1). This was a landmark event in medicine that ushered in unprecedented interest in CT screening for pulmonary malignancies (1). The upshot of this is that incidental findings of small pulmonary ground glass opacities (GGOs) are now increasingly common (2-5). Depending on the consolidation-to-tumor ratio (CTR) on CT imaging, these can be classified into pure GGOs (CTR =0 with no obscuring of vascular markings), and part solid or mixed GGOs $(0<\mathrm{CTR}<1)(6)$. Although guidelines exist to assist in managing these findings (7-12), many unresolved questions and problems remain especially in concern to extent of surgical resection (13).

The Italian Society of Thoracic Surgery (Societa' Italiana Chirurgia Toracica; SICT) recently published the results of a survey of 160 of its members regarding their management of GGOs (14). Even though this represents the views of surgeons in one country only, it is not difficult to imagine that the findings would have been similar in many developed countries around the world. It is therefore worthwhile summarizing their key findings:

* There was good agreement between the respondents on what the definition of a pure GGO was, but less consensus on the definition of a mixed GGO;

* There was good agreement that high-resolution CT should be used for the diagnostic workup of GGOs, but more than $40 \%$ of respondents also supported routine use of PET even for pure GGO even though guidelines do not recommend this;

* There was good agreement that surgical resection was indicated in cases of persistent or growing mixed GGOs after an initial follow-up, but very divided opinion on whether surgery should be offered for persistent pure GGOs;

* There was good agreement that sublobar resection was more viable than a lobectomy for a c-I GGO with a $<50 \%$ solid component, but divergent opinions on whether a wedge or a segmental resection should be performed and on the optimal strategy for lymph nodal dissection;

* In contrast, there was agreement that sublobar resection was "not oncologically comparable" to a lobectomy for a c-I GGO with a $>50 \%$ solid component, and that in such cases radical lymph nodal dissection should be performed;

* Although use of mini-invasive video-assisted surgical procedures for non-small cell lung cancer (NSCLC) was claimed by $96.2 \%$ of respondents, $68 \%$ considered video-assisted thoracic surgery (VATS) to be appropriate only in selected cases of GGOs;

* Although 68\% recommended radiological followup for pure-GGOs measuring $<5 \mathrm{~mm}$, there were divergent opinions regarding scheduling of followup CT scan and regarding for how long to follow-up before discontinuing.

The SICT survey confirms our concerns from our earlier review of GGO management guidelines (13). Namely, that shortcomings in the current guidelines are present, and this 
in turn leads to considerable uncertainties and divergent practices amongst clinicians. The situation is especially acute for surgeons given the paucity of specific surgical recommendations in these guidelines, which were mostly authored primarily by non-surgeons.

However, the SICT survey also shows another glaring gap in our understanding of screening-detected GGO management: there is a complete absence of any mention of multiple GGOs.

Multiple or synchronous GGOs are defined as more than two GGOs found at the same time in a single patient (15-18). Multiple GGOs are an increasingly frequent finding, with around $20-30 \%$ of GGO lesions resected found to be accompanied by multiple other smaller intrapulmonary GGO lesions. Despite this, the surgical management of multiple GGOs detected by screening is even less well defined that for solitary GGOs. For multiple GGOs, no standard algorithms have been established for the selection of which lesions (if any) to be treated, nor for the follow-up of residual nodules that are not treated. Where surgical intervention has been considered, various surgical approaches have been suggested $(19,20)$. Depending on their anatomical location, size, and number, it has been suggested that various combinations of sublobar versus lobar resections, and of one- or two-stage surgery for bilateral lesions may be considered. However, standard algorithms again do not exist.

The main limitation in establishing management protocols for screening-detected multiple GGOs is the relative paucity of clinical evidence for their natural history, diagnosis and treatment. A few themes about these multiple GGOs have emerged and can be summarized thusly:

(I) Overall low risk of change. A study from Korea looked at 73 patients who underwent pulmonary resection for bronchioloalveolar carcinoma, of whom 23 had multiple GGOs seen on pre-operative CT (19). In those 18 patients who did not have all GGO lesions resected, none of the residual GGO lesions progressed in size or solidity on follow-up for a median of 40.3 months. In a study from Japan that included 78 patients with multiple GGOs that were followed up for a median of 45.5 months, interval growth in size was observed in only $37 \%-$ and the majority of these were seen within the first 36 months (21). The authors suggest that the optimal observation period for patients with multiple GGOs is 36 months. A handful of studies have since further suggested that in patients with multiple GGOs, the overall risk of change on follow-up is low and that for multiple subsolid nodules smaller than $6 \mathrm{~mm}$ the likelihood of benign inflammatory disease is higher than malignancy $(22,23)$. Fleischner Society Guidelines therefore recommend $\mathrm{CT}$ at 3-6 months in the first instance for all cases of multiple GGOs (23).

(II) Independent characteristics of each GGO. A number of studies have suggested that the above view of multiple GGOs as being largely 'benign' is perhaps too simplistic. In one series where 46 GGOs were resected from 21 patients, a large variety of pathologies were found-ranging from sclerosing hemangioma, to atypical adenomatous hyperplasia (AAH), to adenocarcinoma in situ (AIS), to minimally invasive adenocarcinoma (MIA), and even to invasive adenocarcinoma (24). Even in this limited series, it is clear that the possibility of frank malignancy cannot be so easily dismissed. In an intriguing study from the Shanghai Pulmonary Hospital, the EGFR mutational profiles in 159 multiple GGO lesions from 78 patients showed great variety (25). Of the 38 paired lesions in patients harbouring EGFR mutation, the discordance rate of EGFR mutation was $92.1 \%$. These results suggest that the individual lesions in a patient with multiple GGOs seem to arise as multiple events, and hence may warrant a more nuanced protocol for management rather than a 'one size fits all' recommendation.

(III) Prognosis dependent on predominant/main lesion. Evidence is emerging that given the independent characteristics of each of the multiple GGOs in a patient, management should be determined by the one 'main' or predominant lesion that carries the worst prognosis. The Fleischner Society suggests that size may be a factor: if at least one of the GGOs is $6 \mathrm{~mm}$ or larger and that persists on follow-up CT at 3-6 months, it is advised to "consider multiple primary adenocarcinomas" (23). A study from Japan suggests that both size and solidity of the main lesion are important (26). If the main GGO lesion is solid-dominant or is larger than $25 \mathrm{~mm}$, it carries worse prognosis (poorer 5 -year overall survival) than if it is GGOdominant and has a size $\leq 25 \mathrm{~mm}$. An International Association for the Study of Lung Cancer (IASLC) subcommittee conducted a systematic review on adenocarcinoma subtypes presenting with multiple GGO lesions on CT (27). Although this review 
focused on patients with histologically proven adenocarcinoma rather than screening-detected multiple GGOs, it arrived at a similar conclusion that prognosis in cases of multifocal ground glass/ lepidic tumors is largely determined by the main lesion with the highest rank in the $\mathrm{T}$ category of the TNM staging system. However, another Japanese study with 246 patients with multiple GGOs found that the solidity of both the main and the 'additional' GGOs influenced survival (28). This study found that solid-dominance in both main and additional GGOs was associated with much worse survival, while GGO-dominance in both was associated with better survival. This result seems to be in contrast to the earlier calls for emphasis to be placed on the main lesion alone.

It is noteworthy that these key themes suggested on the natural history of screening-detected multiple GGOs can be so readily summarized in a few short paragraphs. This illustrates that there is relatively little hard data currently available on which to base robust management algorithms. What evidence exists is also often contradictory—as implied above. In addition, it should also be remembered that radiological assessment of the risk of malignancy in GGOs is often not highly accurate or even consistent $(29,30)$. This potentially further undermines efforts to establish management guidelines based on images from radiological screening alone. Surgical techniques to resect solitary GGO lesions are well described $(31,32)$. However, evidence to guide operative strategy for multiple lesions is less well developed. Questions regarding acceptable extent of lung resection, approach to lymph nodes, whether to perform one- or two-stage surgery for bilateral lesions, and on, appear completely unaddressed.

Clearly, what is currently required today is the generation of more clinical data, but also a thorough analysis and synthesis of what clinical data has already been generated to date. We have previously argued for the need to consider new guidelines on the management of screening-detected solitary GGOs-based on the latest evidence and with practical emphasis on thoracic surgical application (13). The SICT survey has reconfirmed the lack of consensus on the management of pulmonary GGOs amongst surgeons and hence the pressing need for such newer guidelines. The survey has also provided a timely reminder of the large gaps in knowledge surrounding multiple GGOs.

The authors of this article are co-chairs on a new collaboration between the Asia Thoracoscopic Surgery
Education Platform (ATEP), the European Society of Thoracic Surgeons (ESTS), and the European Association for Cardio-Thoracic Surgery (EACTS) respectively. A conjoint task force will now embark on a systematic literature review to build an evidence base regarding management of GGOs with a focus on surgical aspects. It is envisaged that the work of this task force will aim to address the above issues on the management of both solitary and multiple GGOs.

\section{Acknowledgements}

None.

\section{Footnote}

Conflicts of Interest: The authors have no conflicts of interest to declare.

\section{References}

1. Aberle DR, Adams AM, Berg CD, et al. Reduced lungcancer mortality with low-dose computed tomographic screening. N Engl J Med 2011;365:395-409.

2. Church TR, Black WC, Aberle DR, et al. Results of initial low-dose computed tomographic screening for lung cancer. N Engl J Med 2013;368:1980-91.

3. Zhao SJ, Wu N. Early detection of lung cancer: Lowdose computed tomography screening in China. Thoracic Cancer 2015;6:385-9.

4. Nawa T, Nakagawa T, Mizoue T, et al. Low-dose Computed Tomography Screening in Japan. J Thorac Imaging 2015;30:108-14.

5. Yousaf-Khan U, van der Aalst C, de Jong PA, et al. Final screening round of the NELSON lung cancer screening trial: the effect of a 2.5 -year screening interval. Thorax 2017;72:48-56.

6. Hattori A, Matsunaga T, Hayashi T, et al. Prognostic Impact of the Findings on Thin-Section Computed Tomography in Patients with Subcentimeter Non-Small Cell Lung Cancer. J Thorac Oncol 2017;12:954-62.

7. Naidich DP, Bankier AA, MacMahon H, et al. Recommendations for the Management of Subsolid Pulmonary Nodules Detected on CT: A Statement from the Fleischner Society. Radiology 2013;266:304-17.

8. Japanese Society for CT Screening. Guidelines for the management of pulmonary nodules detected by low-dose CT lung cancer screening version 3. Available online: http:// 
www.jscts.org/pdf/guideline/gls3rd_english130621.pdf

9. Gould MK, Donington J, Lynch WR, et al. Evaluation of individuals with pulmonary nodules: when is it lung cancer? Diagnosis and management of lung cancer, 3rd ed: American College of Chest Physicians evidence-based clinical practice guidelines. Chest 2013;143:e93S-120S.

10. National Comprehensive Cancer Network (NCCN) Clinical Practice Guidelines in Oncology. Lung Cancer Screening Version 2.2014. Available online: https://www.trikobe.org/nccn/guideline/lung/english/lung_screening.pdf

11. Jaklitsch MT, Jacobson FL, Austin JH, et al. The American Association for Thoracic Surgery guidelines for lung cancer screening using low-dose computed tomography scans for lung cancer survivors and other high-risk groups. J Thorac Cardiovasc Surg 2012;144:33-8.

12. Callister ME, Baldwin DR, Akram AR, et al. British Thoracic Society guidelines for the investigation and management of pulmonary nodules. Thorax 2015;70:ii1-54.

13. Sihoe AD, Cardillo G. Solitary pulmonary groundglass opacity: is it time for new surgical guidelines? Eur J Cardiothorac Surg 2017;52:848-51.

14. Lococo F, Cusumano G, De Filippis AF, et al. Current Practices in the Management of Pulmonary Ground-Glass Opacities: A Survey of SICT Members. Ann Thorac Surg 2018. [Epub ahead of print].

15. Chang B, Hwang JH, Choi YH, et al. Natural history of pure ground-glass opacity lung nodules detected by lowdose CT scan. Chest 2013;143:172-8.

16. Lee CT. What do we know about ground-glass opacity nodules in the lung? Transl Lung Cancer Res 2015;4:656-9.

17. Moon Y, Sung SW, Lee KY, et al. Pure ground-glass opacity on chest computed tomography: predictive factors for invasive adenocarcinoma. J Thorac Dis 2016;8:1561-70.

18. Pedersen JH, Saghir Z, Winkler Wille MM, et al. Groundglass opacity lung nodules in the era of lung cancer CT screening: radiology, pathology, and clinical management. Oncology (Williston Park) 2016;30:266-74.

19. Kim HK, Choi YS, Kim J, et al. Management of multiple pure ground-glass opacity lesions in patients with bronchioloalveolar carcinoma. J Thorac Oncol 2010;5:206-10.

20. Wang Q, Jiang W, Xi J. Surgery for Pulmonary Multiple Ground Glass Opacities. Zhongguo Fei Ai Za Zhi 2016;19:355-8.

21. Sato Y, Fujimoto D, Morimoto T, et al. Natural history and clinical characteristics of multiple pulmonary nodules with ground glass opacity. Respirology 2017;22:1615-621.

22. Yankelevitz DF, Yip R, Smith JP, et al. CT screening for lung cancer: nonsolid nodules in baseline and annual repeat rounds. Radiology 2015;277:555-64.

23. MacMahon H, Naidich DP, Goo JM, et al. Guidelines for Management of Incidental Pulmonary Nodules Detected on CT Images: From the Fleischner Society 2017. Radiology 2017;284:228-43.

24. Shao G, Ren W, Feng Z, et al. The role of video-assisted thoracoscopic surgery in management of the multiple ground-glass nodules. Indian J Cancer 2015;52:e75-9.

25. Liu M, He WX, Song N, et al. Discrepancy of epidermal growth factor receptor mutation in lung adenocarcinoma presenting as multiple ground-glass opacities. Eur J Cardiothorac Surg 2016;50:909-13.

26. Shimada Y, Saji H, Otani K, et al. Survival of a surgical series of lung cancer patients with synchronous multiple ground-glass opacities, and the management of their residual lesions. Lung Cancer 2015;88:174-80.

27. Detterbeck FC, Marom EM, Arenberg DA, et al. The IASLC Lung Cancer Staging Project: Background Data and Proposals for the Application of TNM Staging Rules to Lung Cancer Presenting as Multiple Nodules with Ground Glass or Lepidic Features or a Pneumonic Type of Involvement in the Forthcoming Eighth Edition of the TNM Classification. J Thorac Oncol 2016;11:666-80.

28. Hattori A, Matsunaga T, Takamochi K, et al. Radiological classification of multiple lung cancers and the prognostic impact based on the presence of a ground glass opacity component on thin section computed tomography. Lung Cancer 2017;113:7-13.

29. Lee SM, Park CM, Goo JM, et al. Invasive pulmonary adenocarcinomas versus preinvasive lesions appearing as ground-glass nodules: differentiation by using CT features. Radiology 2013;268:265-73.

30. Wilshire CL, Louie BE, Manning KA, et al. Radiologic Evaluation of Small Lepidic Adenocarcinomas to Guide Decision Making in Surgical Resection. Ann Thorac Surg 2015;100:979-88.

31. Sihoe AD, Van Schil P. Non-small cell lung cancer: when to offer sublobar resection. Lung Cancer 2014;86:115.

32. Shi Z, Chen C, Jiang S, et al. Uniportal video-assisted thoracic surgery resection of small ground-glass opacities (GGOs) localized with CT-guided placement of microcoils and palpation. J Thorac Dis 2016;8:1837-40.

Cite this article as: Sihoe AD, Petersen RH, Cardillo G. Multiple pulmonary ground glass opacities: is it time for new guidelines? J Thorac Dis 2018;10(11):5970-5973. doi: $10.21037 /$ jtd.2018.10.67 